\title{
Association of visit-to-visit variability in blood pressure with cognitive function in old age: prospective cohort study Sattar professor ${ }^{8}$, J Wouter Jukema professor ${ }^{9}$, Matthias J P van Osch magnetic resonance imaging physicist ${ }^{2}$, Jeroen van der Grond senior neuroradiology researcher ${ }^{2}$, Mark A van Buchem professor ${ }^{2}$, Rudi G J Westendorp professor ${ }^{13}$, Anton J M de Craen senior epidemiologist ${ }^{13}$, Simon P Mooijaart internist and clinical researcher ${ }^{1310}$

\footnotetext{
${ }^{1}$ Department of Gerontology and Geriatrics, Leiden University Medical Center, PO Box 9600, 2300 RC Leiden, Netherlands; ${ }^{2}$ Department of Radiology, Leiden University Medical Center, Netherlands; ${ }^{3}$ Netherlands Consortium for Healthy Ageing, Leiden, Netherlands; ${ }^{4}$ Department of Psychiatry, Leiden University Medical Center, Netherlands; ${ }^{5}$ Academic Section of Geriatrics, Faculty of Medicine, University of Glasgow, UK; ${ }^{6}$ Robertson Center for Biostatistics, University of Glasgow, UK; ${ }^{7}$ Department of Pharmacology and Therapeutics, University College Cork, Ireland; ${ }^{8}$ British Heart Foundation, Glasgow Cardiovascular Research Centre, University of Glasgow, UK; ${ }^{9}$ Department of Cardiology, Leiden University Medical Center, Leiden, Netherlands; ${ }^{10}$ Institute for Evidence-Based Medicine in Old age, Leiden, the Netherlands
}

\begin{abstract}
Objective To investigate the association between visit-to-visit variability in blood pressure and cognitive function in old age (>70 years).

Design Prospective cohort study.

Setting PROSPER (PROspective Study of Pravastatin in the Elderly at Risk) study, a collaboration between centres in Ireland, Scotland, and the Netherlands.

Participants 5461 participants, mean age 75.3 years, who were at risk of cardiovascular disease. Blood pressure was measured every three months during an average of 3.2 years. Visit-to-visit variability in blood pressure was defined as the standard deviation of blood pressure measurements between visits.
\end{abstract}

Main outcome measures Four domains of cognitive function, testing selective attention, processing speed, and immediate and delayed memory. In a magnetic resonance imaging substudy of 553 participants, structural brain volumes, cerebral microbleeds, infarcts, and white matter hyperintensities were measured.

Results Participants with higher visit-to-visit variability in systolic blood pressure had worse performance on all cognitive tests: attention (mean difference high versus low thirds) 3.08 seconds ( $95 \%$ confidence interval 0.85 to 5.31 ), processing speed -1.16 digits coded ( $95 \%$ confidence interval -1.69 to -0.63$)$, immediate memory -0.27 pictures remembered ( $95 \%$ confidence interval -0.41 to -0.13 ), and delayed memory -0.30 pictures remembered ( $95 \%$ confidence interval -0.49 to -0.11 ). Furthermore, higher variability in both systolic and diastolic blood pressure was associated with lower hippocampal volume and cortical infarcts, and higher variability in diastolic blood pressure was associated with cerebral microbleeds (all $\mathrm{P}<0.05$ ). All associations were adjusted for average blood pressure and cardiovascular risk factors.

Conclusion Higher visit-to-visit variability in blood pressure independent of average blood pressure was associated with impaired cognitive function in old age.

\section{Introduction}

Visit-to-visit variability in blood pressure independent of average blood pressure is related to cerebrovascular damage. ${ }^{1}$ It has been shown that higher blood pressure variability increases the risk of stroke and that antihypertensives, which decrease both variability in blood pressure and mean blood pressure, more effectively reduce the risk of stroke. ${ }^{2}$ In addition, observational studies have shown associations of variability in blood pressure, independent of average blood pressure, with 
white matter hyperintensities, carotid artery intima media thickness, and atherosclerosis in older people ( $\geq 55$ years). ${ }^{3-5}$

The relation between increased variability in blood pressure and end organ damage is well established. ${ }^{6}$ Recent evidence indicates that higher visit-to-visit variability is linked with microvascular damage, endothelial injury, and disturbances in vascular smooth muscle functioning. ${ }^{78}$ Indicators of cerebral small vessel disease, including white matter hyperintensities, cortical microinfarcts, and cerebral microbleeds are implicated in the pathogenesis of cognitive impairment. ${ }^{9-11}$ Several pathological, observational, and experimental studies have shown that disruption of the blood-brain barrier due to microvascular damage results in neuronal injury and accelerates neuronal loss and brain atrophy. ${ }^{12}$ Hence higher variability in blood pressure might potentially lead to cognitive impairment through changes in the brain structures and development of cerebral small vessel disease.

We investigated the association of variability in blood pressure between visits independent of average blood pressure with cognitive function in older participants ( $>70$ years) at high risk of cardiovascular disease. Additionally, we investigated possible explanations behind this association in a magnetic resonance imaging substudy.

\section{Methods}

\section{Study design and participants}

The data in this study were obtained from PROSPER (The PROspective Study of Pravastatin in the Elderly at Risk), a randomised, double blind, placebo controlled trial designed to investigate the effect of pravastatin in the prevention of vascular events in elderly people with pre-existing, or risk factors for, cardiovascular disease. This trial included 5804 people aged 70-82 years who were enrolled from three collaborating centres in Ireland, Scotland, and the Netherlands. Approximately 50\% of the participants showed evidence of cardiovascular disease, including stable angina, intermittent claudication, stroke (the type, haemorrhagic or ischaemic, was unknown), transient ischaemic attack, myocardial infarction, and vascular surgery. The rest of the participants had one or more major cardiovascular risk factors, defined as hypertension, cigarette smoking, or diabetes mellitus. The primary outcome of the study was the combined endpoint of definite or suspected death from coronary heart disease, non-fatal myocardial infarction, and fatal or non-fatal stroke during a mean follow-up of 3.2 years. In the present study we included 5461 participants for whom data on variability in blood pressure and cognitive function were available. Additionally, participants from the Netherlands were invited to participate in a magnetic resonance imaging substudy. Participants were included from both the pravastatin and the placebo groups as we previously reported that treatment with pravastatin did not influence cognitive function, structural brain volumes, or indicators of cerebral small vessel disease. ${ }^{13-15}$

\section{Blood pressure measurements}

We measured systolic and diastolic blood pressure at baseline and every three months. Blood pressure was measured with participants in the sitting position and using a fully automatic electronic sphygmomanometer (Omron M4, Kyoto, Japan). All measurements were performed in the same clinical setting. In the analyses we used the average values of these blood pressure measurements. We defined visit-to-visit variability in blood pressure as the standard deviation of blood pressure measurements during the study period. We report the variability in blood pressure using only the standard deviation. Variance and coefficient of variation, which are two other measures of variability, are strongly correlated with the standard deviation (see supplementary table S-1) and they showed similar associations with cognitive and magnetic resonance imaging outcomes (data not shown).

\section{Cognitive function}

The mini-mental state examination was used to evaluate global cognitive function at baseline; to exclude participants with poor cognitive function at baseline we used a cut-off score of 24 points or more (out of 30) as an inclusion criterion. In the present study we used data on cognitive function assessed at the end of the study, after a mean follow-up of 3.2 years, by a cognitive test battery consisting of four different tests. The Stroop colour and word test was used to assess selective attention and reaction time. The participants were asked to read the name of a colour, which appeared in a colour different from that being named. The outcome variable was the total number of seconds to complete the test; a higher score indicating worse performance. General cognitive speed was tested by the letter-digit coding test. The participants had to match certain digits with letters according to a provided key. The outcome variable was the total number of correct entries in 60 seconds, with higher scores indicating better performance. The picture-word learning test was used to assess immediate and delayed memory. The participants were shown 15 pictures and were then asked to recall as many pictures as possible in three trials. After 20 minutes they were asked to repeat the test to measure their delayed recall. The outcome variable was the accumulated number of correct recalled pictures, immediately and after 20 minutes, with higher scores indicating better performance. Detailed descriptions of the cognitive tests and the procedures have been published previously. ${ }^{16}$

\section{Magnetic resonance imaging substudy}

Overall, 646 of the 1100 Dutch participants in the PROSPER study consented to participate in the magnetic resonance imaging substudy. Forty of the 646 original study participants died during the follow-up period. Magnetic resonance imaging was performed at the end of the follow-up period in the remaining 606 participants. Data on visit-to-visit variability in blood pressure and magnetic resonance imaging were available for 553 participants. Details of individual variables of magnetic resonance imaging scanning have been published previously. ${ }^{13}$

All imaging was performed on a magnetic resonance system operating at a field strength of 1.5 Tesla (Philips Medical Systems, Best, Netherlands). We used the SIENAX technique to calculate grey and white matter volumes. In short, SIENAX starts by extracting brain and skull images from input data for the whole head. The brain image is then affine registered to Montreal Neurological Institute 152 space (by using the skull image to determine the registration scaling), done primarily to obtain the volumetric scaling factor to be used as normalisation for head size. Next we carried out tissue type segmentation with partial volume estimation to calculate the total volume of brain tissue (including separate estimates of volumes of grey matter, white matter, peripheral grey matter, and ventricular cerebrospinal fluid). ${ }^{17}$ The algorithm FIRST (the Oxford Centre for Functional MRI of the Brain's (FMRIB) integrated registration and segmentation tool) was applied to estimate the volume of hippocampus. In addition, we estimated the volume of six other subcortical regions, including nucleus accumbens, globus pallidus, amygdala, putamen, caudate nucleus, and thalamus. FIRST is part of FSL (FMRIB's software library) and performs both registration and segmentation of the mentioned subcortical regions. ${ }^{18}$ To assess cerebral microbleeds, 
two experienced raters blinded to the participants' clinical history read all the magnetic resonance imaging scans in consensus. Cerebral microbleeds were defined as focal areas of signal loss on T2 weighted gradient echo pulse sequence ("blooming effect") that were invisible or smaller on T2 weighted magnetic resonance imaging. ${ }^{19}$. For each participant we recorded the number and location (cortical, subcortical, and infratentorial) of the cerebral microbleeds. Segmentation of white matter hyperintensities volume was performed automatically using software for Neuro-Image Processing in Experimental Research (SNIPER), an in-house developed program for image processing. ${ }^{20}$ This segmentation was based on the $\mathrm{T} 2$ weighted and fluid attenuated inversion recovery (FLAIR) images. Cerebral infarcts were defined as parenchymal defects seen on FLAIR images with the same signal intensity as cerebrospinal fluid and a surrounding rim of high signal intensity after a vascular distribution.

\section{Personal and clinical characteristics}

We recorded the personal, medical, and anthropometric data of the participants at baseline. A fasting venous blood sample was taken for biochemical and haematological assessment. Western blotting was used on the plasma samples to determine apolipoprotein E epsilon 2/3/4 phenotype..$^{21}$

\section{Statistical analysis}

Characteristics of the study participants are reported as mean (standard deviation) for continuous variables and frequency (percentage) for categorical variables. We used Pearson's correlation coefficient to calculate the correlation between variability in blood pressure and average blood pressure. Linear regression models were used to assess the association of variability in blood pressure and average blood pressure with cognitive function. Dependent variables were the mean scores of the cognitive tests. In the tables these scores are presented in thirds of systolic and diastolic blood pressure and blood pressure variability. In the magnetic resonance imaging substudy, we used logistic regression models to estimate the odds ratio and $95 \%$ confidence interval of the presence of microbleeds or infarcts in different thirds of blood pressure variability as well as average blood pressure. We used multivariable linear regression models to test the association between blood pressure variability and average blood pressure with volume of white matter hyperintensities and structural brain volumes. $\mathrm{P}$ values in all analyses were calculated using systolic and diastolic blood pressure variability as continuous variables.

We performed our analyses in three steps. In the first step, we carried out crude analyses, in which we only adjusted for cognitive test version where appropriate. In the second step, we added age, sex, education, and country as covariates to investigate the potential influence of these factors on the associations (model 1). In the final model (model 2), we further adjusted the analyses for the following potential confounders: cardiovascular diseases and risk factors (history of vascular disease, history of hypertension, history of diabetes mellitus, smoking status, cholesterol levels, body mass index), average blood pressure, statin treatment, and apolipoprotein E genotype. We adjusted the analyses of systolic blood pressure variability with cognitive function and magnetic resonance imaging outcomes for average systolic blood pressure. The analyses of variability in diastolic blood pressure with cognitive function and magnetic resonance imaging outcomes were adjusted for average diastolic blood pressure. Since the associations did not essentially change in different models, results of the second model are presented in the manuscript and results from the other models are presented in the supplementary file (tables S-2 and S-3). All analyses were performed using SPSS software (version 20.0.0).

\section{Results}

Table $1 \Downarrow$ shows the characteristics of the participants in the whole group and in the magnetic resonance imaging substudy. Blood pressure was measured in an average number of 12.7 visits in the whole group and 12.9 visits in the magnetic resonance imaging substudy. Average systolic and diastolic blood pressure over the period of blood pressure measurements were $153.1 \mathrm{~mm} \mathrm{Hg}$ and $82.5 \mathrm{~mm} \mathrm{Hg}$, respectively. The corresponding mean standard deviation values during this period were $14.8 \mathrm{~mm} \mathrm{Hg}$ and $7.1 \mathrm{~mm} \mathrm{Hg}$.

There was a weak but significant correlation between average systolic blood pressure and standard deviation of systolic blood pressure measurements $(\mathrm{r}=0.20, \mathrm{P}<0.001)$. Similarly, the average diastolic blood pressure was weakly but significantly correlated with the standard deviation of diastolic blood pressure measurements $(\mathrm{r}=0.12, \mathrm{P}<0.001)$.

Table $2 \Downarrow$ shows the association of visit-to-visit variability in systolic and diastolic blood pressure with cognitive function. Higher variability was associated with worse performance on the Stroop test (both $\mathrm{P}<0.001$ ), letter-digit coding test (both $\mathrm{P}<0.001$ ), immediate picture-word learning test (both $\mathrm{P}<0.001$ ), and delayed picture-word learning test (both $\mathrm{P}=0.001$ ). All associations were independent of average blood pressure and cardiovascular diseases and risk factors, as all analyses were adjusted for these factors. The figure $\downarrow$ presents the mean cognitive scores (95\% confidence intervals) in each third of systolic and diastolic blood pressure variability. Data on the association of blood pressure variability with cognitive function from crude and minimally adjusted models are shown in the supplementary file (tables S-2 and S-3). Furthermore, we found a significant association of higher average systolic and diastolic blood pressure with worse performance in different domains of cognitive function (all $\mathrm{P}<0.05$ ), except for the association between higher average systolic blood pressure and performance on the picture-word learning tests $(\mathrm{P}>0.05)$ (see supplementary table S-4).

Table $3 \Downarrow$ shows the association of visit-to-visit variability in systolic and diastolic blood pressure with structural brain volumes. Higher variability was associated with lower hippocampal volume (both $\mathrm{P}=0.01$ ). There was no association between blood pressure variability and volume of the other brain structures (all $\mathrm{P}>0.05$ ), except for the association between higher variability in systolic blood pressure and lower amygdala and putamen volumes (both $\mathrm{P}=0.04$ ). Analyses were adjusted for average systolic and diastolic blood pressures, which themselves were not associated with structural brain volumes (all $\mathrm{P}>0.05$ ) (see supplementary table S-5).

Table $4 \Downarrow$ shows the association between visit-to-visit variability in blood pressure and cerebral microbleeds, infarcts, and white matter hyperintensities. Higher variability in systolic and diastolic blood pressure was associated with a higher risk of cortical infarcts (both $\mathrm{P}=0.02$ ). Prevalence of cortical infarcts in participants with low, middle, and high variability in systolic blood pressure was $9.2 \%, 12.0 \%$, and $16.2 \%$, respectively. Prevalence of cortical infarcts in participants with low, middle, and high variability in diastolic blood pressure was $7.9 \%, 13.3 \%$, and $16.2 \%$, respectively. Furthermore, higher variability in diastolic blood pressure was associated with a higher risk of all types of microbleeds $(\mathrm{P}=0.01)$ as well as subcortical microbleeds $(\mathrm{P}=0.004)$. Prevalence of microbleeds in participants with low, 
middle, and high variability in systolic blood pressure was $21.2 \%, 23.9 \%$, and $28.4 \%$, respectively. Prevalence of cortical infarcts in participants with low, middle, and high variability in diastolic blood pressure was $17.8 \%, 27.5 \%$, and $28.9 \%$, respectively. Variability in systolic and diastolic blood pressure was not associated with white matter hyperintensities (both $\mathrm{P}>0.05$ ). We found no association of average systolic and diastolic blood pressure with cerebral microbleeds, infarcts, and white matter hyperintensities (all $\mathrm{P}>0.05$ ) (see supplementary table S-6).

We performed four sensitivity analyses to explore whether the association of visit-to-visit variability in blood pressure with the studied outcomes could be affected by participants with a history of clinical stroke or transient ischaemic attack $(n=606)$ and cardiovascular disease $(n=2404)$, participants with new or a change in antihypertensive therapy during the study period $(n=2733)$, participants who developed vascular events $(n=872)$ or arrhythmia $(\mathrm{n}=506)$ during the study period, and participants with a high average blood pressure (defined as average systolic blood pressure of $\geq 140 \mathrm{~mm} \mathrm{Hg}$ and diastolic blood pressure of $\geq 80 \mathrm{~mm} \mathrm{Hg}$ during the study period) $(\mathrm{n}=830)$. These sensitivity analyses showed that the results did not materially change. In an overall sensitivity analysis we excluded all participants with the aforementioned conditions $(n=4654)$ and the results remained essentially unchanged (data not shown).

\section{Discussion}

Higher visit-to-visit variability in systolic and diastolic blood pressure was associated with worse performance in different domains of cognitive function and lower hippocampal volume and risk of cortical infarcts. Higher variability in diastolic blood pressure was associated with risk of cerebral microbleeds. These associations were independent of various cardiovascular risk factors, in particular average systolic and diastolic blood pressures.

Although hypertension is a well established risk factor for cardiovascular diseases, ${ }^{22}$ increasing evidence indicates that the predictive value of conventional blood pressure measurement for cardiovascular diseases attenuates with increasing age. ${ }^{23}{ }^{24}$ Recent studies have shown that higher visit-to-visit variability in blood pressure increases the risk of cardiovascular events, ${ }^{25}$ stroke, ${ }^{11} 24$ and carotid artery atherosclerosis ${ }^{26}$ in older people, independent of average blood pressure. Given the link between neurovascular dysfunction and cognitive impairment, ${ }^{27}$ a recent study on 201 elderly participants (mean age 79.9 years) at high risk of cardiovascular disease showed that high visit-to-visit variability in blood pressure during 12 months was associated with worse performance in the mini-mental state examination and global deterioration scale. ${ }^{28}$ Consistent with this finding, by using a population of over 5000 participants and over three years of blood pressure measurements, we showed that high visit-to-visit variability in both systolic and diastolic blood pressure was associated with worse performance in different domains of cognitive function, including selective attention, processing speed, immediate verbal memory, and delayed memory.

The magnitude of associations in this study, reflected as differences in cognitive scores between top and bottom thirds of variability in systolic and diastolic blood pressure, are comparable with the observed differences in cognitive function between groups of apolipoprotein $\mathrm{E}$ genotype on cognitive function. ${ }^{29}$ The apolipoprotein E4 genotype is a well recognised risk factor for the development of dementia in later life and it has been shown that people who carry this risk factor have a four times higher risk of developing late onset Alzheimer's disease. ${ }^{30}$ Similar differences in cognitive test scores in apolipoprotein E groups and variability in blood pressure implies that the observed associations can be considered clinically relevant.

Different explanations can be proposed for the observed association between high visit-to-visit variability in blood pressure and impaired cognitive function. Firstly, both blood pressure variability and cognitive impairment could stem from a common cause, without themselves being causally related. Cardiovascular risk factors are the most likely candidate. ${ }^{31}$ Nevertheless, we reported our analyses adjusted for different cardiovascular risk factors and we performed a sensitivity analysis, by separately excluding those with a history of cardiovascular diseases. This did not change our estimates, although we accept that residual confounding could remain from unmeasured risk factors for cardiovascular disease. As a second explanation, high visit-to-visit variability in blood pressure might reflect a long term haemodynamic instability in the systemic circulation that puts stress on the vascular endothelium. ${ }^{72}$ This haemodynamic stress may lead to endothelial dysfunction and microvascular damage with consequent alterations in brain structure and function..$^{33}$ Thirdly, exaggerated fluctuations in systemic blood pressure could result in repeated episodes of cerebral hypoperfusion causing neuronal injury and cell death, particularly in vulnerable brain regions such as the hippocampus. ${ }^{4}$ In line with latter explanations, we found that higher visit-to-visit variability in blood pressure is related to lower hippocampal volume and the presence of cerebral microbleeds and cortical infarcts. Given the well described association of hippocampal atrophy ${ }^{34}$ and cerebral small vessel disease ${ }^{10}$ with cognitive impairment, our findings may suggest that decreased hippocampal volume, cerebral microbleeds, and cortical infarcts are potential pathogenic mechanisms behind the association between variability in blood pressure and cognitive impairment.

Current evidence on the association of variability in blood pressure with structural brain damage and cerebral small vessel disease mainly comes from studies that focused on variability in ambulatory blood pressure rather than visit-to-visit variability. These studies showed that higher variability in ambulatory blood pressure is associated with brain atrophy and white matter lesions. ${ }^{35-37}$ In the present study, we only observed the association of visit-to-visit variability in blood pressure with lower hippocampal volume, cerebral microbleeds, and cortical infarcts. This might imply that different measures of blood pressure variability carry different predictive values for brain outcomes. ${ }^{24}$ Data on the association between visit-to-visit variability in blood pressure and manifestations of small vessel diseases are scarce. Consistent with our findings, a recent study showed that higher visit-to-visit variability in blood pressure in people with a history of ischaemic stroke was associated with progression of cerebral microbleeds but not with white matter lesions. ${ }^{38}$ It is, however, still unclear whether higher variability in blood pressure is a cause or consequence of brain disease. It has been suggested that higher variability itself could originate from previously established brain diseases disturbing central autonomic control. ${ }^{39}$ While clinical trials have shown conflicting findings on the benefit of antihypertensive therapy on reducing the risk of dementia, calcium channel blockers, the most effective drug class to reduce variability in blood pressure, ${ }^{40}$ showed significant efficacy in lowering the risk of vascular cognitive impairment. ${ }^{41}$ This might highlight potential clinical implications of agents reducing blood pressure variability in lowering the risk of brain vascular disease and cognitive impairment in old age. 
Collectively, we are not able to make a causal inference from our observation, and future long term investigations are warranted to examine whether strategies to reduce variability in blood pressure can effectively decrease the risk of cognitive impairment as well as of brain vascular disease.

\section{Strengths and limitations of this study}

The major strengths of this study include a large sample size and application of an extended standardised cognitive test battery to assess cognitive function. In addition, availability of neuroimaging data provided us with a unique opportunity to investigate potential biological pathways linked to the association between variability in blood pressure and cognitive function. However, this study has certain limitations. Firstly, we included elderly participants at risk of cardiovascular diseases with relatively preserved cognitive function (mini-mental state examination $\geq 24$ points), which might limit the extrapolation of our findings to a general elderly population. However, this restriction has possibly resulted in a homogeneous study population who are among the main target groups for preventing cognitive decline. ${ }^{42}$ Secondly, the outcomes of this study were evaluated at one time point, and long term longitudinal studies are needed to test whether lowering variability in blood pressure could lead to decelerated cognitive decline and lower the burden of brain diseases. Thirdly, owing to the limited number of participants in the magnetic resonance imaging substudy, we had limited power in several outcome measures. This means that the absence of significant associations for several outcome measures should be interpreted with caution. There are reports indicating that higher visit-to-visit variability in blood pressure is related to a higher risk of stroke and cerebrovascular damage, ${ }^{1}$ however, the exact mechanisms behind these associations are still unclear. This problem needs to be addressed in future magnetic resonance imaging studies with larger numbers of participants. Fourthly, although we adjusted our analyses for different potential confounding factors, some other confounders may exist that we did not consider in our analyses. Future studies investigating the determinants of visit-to-visit variability in blood pressure might help to understand better the association between variability in blood pressure and neurocognitive outcomes.

\section{Conclusions}

In conclusion, our findings suggest that higher visit-to-visit variability in blood pressure independent of average blood pressure is associated with worse cognitive performance in older people at high risk of cardiovascular disease. Changes in hippocampal volume and occurrence of cortical infarcts and cerebral microbleeds might be candidate pathogenic mechanisms behind this association. This observation merits further interventional studies to determine whether reducing variability in blood pressure can decrease the risk of cognitive impairment in old age.

We thank Stella Trompet for her kind collaboration in data analysis. Contributors: BS and LWW contributed equally to the work. They formulated the research question, analysed and interpreted the data, and drafted the manuscript. JCF-D analysed and interpreted the data. DJS, IF, BMB, NS, and JWJ designed the study and revised the manuscript. MJPvO, JvdG interpreted the data and revised the manuscript. MAvB designed the study and interpreted the data. RGJW designed the study, interpreted the data, and revised the draft. AJMdC and SPM formulated the research question, interpreted the data, and revised the manuscript. SPM is guarantor.
Funding: The original PROSPER clinical trial was funded by an investigator initiated grant from Bristol-Myers Squibb, USA. However, the company had no involvement in the formulation of hypotheses, analysis of the data, or in any aspect of the preparation of this manuscript.

Competing interests: All authors have completed the ICMJE uniform disclosure form at www.icmje.org/coi_disclosure.pdf and declare: no support from any organisation for the submitted work; no financial relationships with any organisations that might have an interest in the submitted work in the previous three years; no other relationships or activities that could appear to have influenced the submitted work.

Ethical approval: This study was approved by the institutional ethics committees of the three collaborating centers. All participants gave written informed consent.

Data sharing: No additional data available.

1 Rothwell PM, Howard SC, Dolan E, O'Brien E, Dobson JE, Dahlof B, et al. Prognostic significance of visit-to-visit variability, maximum systolic blood pressure, and episodic hypertension. Lancet 2010;375:895-905

2 Rothwell PM, Howard SC, Dolan E, O'Brien E, Dobson JE, Dahlof B, et al. Effects of beta blockers and calcium-channel blockers on within-individual variability in blood pressure and risk of stroke. Lancet Neurol 2010;9:469-80.

3 Gunstad J, Cohen RA, Tate DF, Paul RH, Poppas A, Hoth K, et al. Blood pressure variability and white matter hyperintensities in older adults with cardiovascular disease. Blood Press 2005:14:353-8.

4 Brickman AM, Reitz C, Luchsinger JA, Manly JJ, Schupf N, Muraskin J, et al. Long-term blood pressure fluctuation and cerebrovascular disease in an elderly cohort. Arch Neurol 2010;67:564-9.

5 Havlik RJ, Foley DJ, Sayer B, Masaki K, White L, Launer LJ. Variability in midlife systolic blood pressure is related to late-life brain white matter lesions: the Honolulu-Asia Aging study. Stroke 2002;33:26-30.

6 Mancia G, Parati G. The role of blood pressure variability in end-organ damage. $J$ Hypertens Supp/ 2003;21:S17-23.

7 Diaz KM, Veerabhadrappa P, Kashem MA, Feairheller DL, Sturgeon KM, Williamson ST, et al. Relationship of visit-to-visit and ambulatory blood pressure variability to vascula function in African Americans. Hypertens Res 2012;35:55-61.

8 Diaz KM, Veerabhadrappa P, Kashem MA, Thakkar SR, Feairheller DL, Sturgeon KM, et al. Visit-to-visit and 24-h blood pressure variability: association with endothelial and smooth muscle function in African Americans. J Hum Hypertens 2013; published online 25 Apr.

9 Van Es AC, van der Grond J, de Craen AJ, Westendorp RG, Bollen EL, Blauw GJ, et al. Cerebral microbleeds and cognitive functioning in the PROSPER study. Neurology 2011;77:1446-52.

10 Pantoni L. Cerebral small vessel disease: from pathogenesis and clinical characteristics to therapeutic challenges. Lancet Neurol 2010;9:689-701.

11 Kovari E, Gold G, Herrmann FR, Canuto A, Hof PR, Michel JP, et al. Cortical microinfarcts and demyelination significantly affect cognition in brain aging. Stroke 2004;35:410-4.

12 Wardlaw JM, Sandercock PA, Dennis MS, Starr J. Is breakdown of the blood-brain barrier responsible for lacunar stroke, leukoaraiosis, and dementia? Stroke 2003:34:806-12.

13 Ten Dam VH, Box FM, de Craen AJ, van den Heuvel DM, Bollen EL, Murray HM, et al. Lack of effect of pravastatin on cerebral blood flow or parenchymal volume loss in elderly at risk for vascular disease. Stroke 2005;36:1633-6.

14 Ten Dam VH, van den Heuvel DM, van Buchem MA, Westendorp RG, Bollen EL, Ford I, et al. Effect of pravastatin on cerebral infarcts and white matter lesions. Neurology 2005;64:1807-9.

15 Trompet S, van Vliet P, de Craen AJ, Jolles J, Buckley BM, Murphy MB, et al. Pravastatin and cognitive function in the elderly. Results of the PROSPER study. J Neurol 2010;257:85-90

16 Houx PJ, Shepherd J, Blauw GJ, Murphy MB, Ford I, Bollen EL, et al. Testing cognitive function in elderly populations: the PROSPER study. PROspective Study of Pravastatin in the Elderly at Risk. J Neurol Neurosurg Psychiatry 2002;73:385-9.

17 Smith SM, Jenkinson M, Woolrich MW, Beckmann CF, Behrens TE, Johansen-Berg $\mathrm{H}$, et al. Advances in functional and structural MR image analysis and implementation as FSL. Neurolmage 2004;23(Suppl 1):S208-19.

18 Widya RL, de Roos A, Trompet S, de Craen AJ, Westendorp RG, Smit JW, et al. Increased amygdalar and hippocampal volumes in elderly obese individuals with or at risk of cardiovascular disease. Am J Clin Nutr 2011;93:1190-5.

19 Altmann-Schneider I, Trompet S, de Craen AJ, van Es AC, Jukema JW, Stott DJ, et al. Cerebral microbleeds are predictive of mortality in the elderly. Stroke 2011;42:638-44.

20 Admiraal-Behloul F, van den Heuvel DM, Olofsen $\mathrm{H}$, van Osch MJ, van der Grond J, van Buchem MA, et al. Fully automatic segmentation of white matter hyperintensities in MR images of the elderly. Neurolmage 2005;28:607-17.

21 Havekes LM, de Knijf P, Beisiegel U, Havinga J, Smit M, Klasen E. A rapid micromethod for apolipoprotein E phenotyping directly in serum. J Lipid Res 1987:28:455-63.

22 Olindo S, Cabre P, Deschamps R, Chatot-Henry C, Rene-Corail P, Fournerie P, et al. Acute stroke in the very elderly: epidemiological features, stroke subtypes, management, and outcome in Martinique, French West Indies. Stroke 2003;34:1593-7.

23 Lewington S, Clarke R, Qizilbash N, Peto R, Collins R. Age-specific relevance of usual blood pressure to vascular mortality: a meta-analysis of individual data for one million adults in 61 prospective studies. Lancet 2002;360:1903-13.

24 Rothwell PM. Limitations of the usual blood-pressure hypothesis and importance of variability, instability, and episodic hypertension. Lancet 2010;375:938-48.

25 Eguchi K, Hoshide S, Schwartz JE, Shimada K, Kario K. Visit-to-visit and ambulatory blood pressure variability as predictors of incident cardiovascular events in patients with hypertension. Am J Hypertens 2012;25:962-8.

26 Dellasega C, Morris D. The MMSE to assess the cognitive state of elders. Mini-Menta State Examination. J Neurosci Nurs 1993;25:147-52. 


\section{What is already known on this topic}

Increased variability in blood pressure is an independent risk factor for cardiovascular events

Participants with greater visit-to-visit variability in blood pressure are at a higher risk for developing silent and clinically evident

cerebrovascular damage

\section{What this study adds}

Higher visit-to-visit variability in blood pressure independent of average blood pressure is associated with impaired cognitive performance in old age

Cerebral microbleeds, cortical infarcts, and changes in hippocampal volume might be candidate pathogenic mechanisms

27 Kim YS, Immink RV, Stok WJ, Karemaker JM, Secher NH, van Lieshout JJ. Dynamic cerebral autoregulatory capacity is affected early in Type 2 diabetes. Clin Sci (Lond) 2008;115:255-62.

28 Nagai M, Hoshide S, Ishikawa J, Shimada K, Kario K. Visit-to-visit blood pressure variations: new independent determinants for cognitive function in the elderly at high risk of cardiovascular disease. J Hypertens 2012:30:1556-63.

29 Packard CJ, Westendorp RG, Stott DJ, Caslake MJ, Murray HM, Shepherd J, et al. Association between apolipoprotein E4 and cognitive decline in elderly adults. J Am Geriatr Soc 2007;55:1777-85

30 Sadigh-Eteghad S, Talebi M, Farhoudi M. Association of apolipoprotein E epsilon 4 allele with sporadic late onset Alzheimer's disease. A meta-analysis. Neurosciences (Riyadh) 2012;17:321-6.

31 Parati G, Lantelme P. Blood pressure variability, target organ damage and cardiovascular events. J Hypertens 2002;20:1725-9.

32 Kim KI, Lee JH, Chang HJ, Cho YS, Youn TJ, Chung WY, et al. Association between blood pressure variability and inflammatory marker in hypertensive patients. Circ $J$ 2008;72:293-8.

33 Cecchi E, Giglioli C, Valente S, Lazzeri C, Gensini GF, Abbate R, et al. Role of hemodynamic shear stress in cardiovascular disease. Atherosclerosis 2011:214:249-56.

34 Barnes J, Bartlett JW, van de Pol LA, Loy CT, Scahill RI, Frost C, et al. A meta-analysis of hippocampal atrophy rates in Alzheimer's disease. Neurobiol Aging 2009;30:1711-23.

35 Goldstein IB, Bartzokis G, Guthrie D, Shapiro D. Ambulatory blood pressure and the brain: a 5-year follow-up. Neurology 2005;64:1846-52.

36 Goldstein IB, Bartzokis G, Guthrie D, Shapiro D. Ambulatory blood pressure and brain atrophy in the healthy elderly. Neurology 2002;59:713-9.

37 Gomez-Angelats E, de La Sierra A, Sierra C, Parati G, Mancia G, Coca A. Blood pressure variability and silent cerebral damage in essential hypertension. Am J Hypertens 2004;17:696-700.
38 Liu W, Liu R, Sun W, Peng $Q$, Zhang W, Xu E, et al. Different impacts of blood pressure variability on the progression of cerebral microbleeds and white matter lesions. Stroke 2012;43:2916-22.

39 Kario K, Motai K, Mitsuhashi T, Suzuki T, Nakagawa Y, Ikeda U, et al. Autonomic nervous system dysfunction in elderly hypertensive patients with abnormal diurnal blood pressure variation: relation to silent cerebrovascular disease. Hypertension 1997:30:1504-10.

40 Webb AJ, Fischer U, Mehta Z, Rothwell PM. Effects of antihypertensive-drug class on interindividual variation in blood pressure and risk of stroke: a systematic review and meta-analysis. Lancet 2010;375:906-15.

41 Lopez-Arrieta JM, Birks J. Nimodipine for primary degenerative, mixed and vascular dementia. Cochrane Database Syst Rev 2002(3):CD000147.

42 Alagiakrishnan $\mathrm{K}, \mathrm{McCracken} \mathrm{P}$, Feldman $\mathrm{H}$. Treating vascular risk factors and maintaining vascular health: is this the way towards successful cognitive ageing and preventing cognitive decline? Postgrad Med J 2006;82:101-5.

Accepted: 12 July 2013

\section{Cite this as: BMJ 2013:347:f4600}

This is an Open Access article distributed in accordance with the Creative Commons Attribution Non Commercial (CC BY-NC 3.0) license, which permits others to distribute, remix, adapt, build upon this work non-commercially, and license their derivative works on different terms, provided the original work is properly cited and the use is non-commercial. See: http://creativecommons.org/licenses/by-nc/3.0/. 


\section{Tables}

Table 1 | Characteristics of study participants in whole group and magnetic resonance imaging (MRI) substudy. Values are means (standard deviations) unless stated otherwise

\begin{tabular}{|c|c|c|}
\hline Characteristics & Whole group $(n=5461)$ & MRI substudy $(n=553)$ \\
\hline No of visits & $12.7(2.4)$ & $12.9(1.5)$ \\
\hline Age (years) & $75.3(3.3)$ & $74.9(3.2)$ \\
\hline No (\%) women & $2822(51.7)$ & $241(43.6)$ \\
\hline Age left school (years) & $15.1(2.1)$ & $15.5(2.9)$ \\
\hline \multicolumn{3}{|l|}{ No (\%) with vascular risk factors: } \\
\hline History of hypertension & 3399 (62.2) & $341(63.1)$ \\
\hline History of diabetes mellitus & $576(10.5)$ & $91(16.5)$ \\
\hline History of stroke or transient ischaemic attack & $606(11.1)$ & $89(16.1)$ \\
\hline History of myocardial infarction & $714(13.1)$ & $67(12.1)$ \\
\hline History of vascular disease & $2404(44.0)$ & $240(43.4)$ \\
\hline Current smoker & $1433(26.2)$ & $115(20.8)$ \\
\hline Body mass index & $26.9(4.2)$ & $26.7(3.6)$ \\
\hline Total cholesterol level & $5.7(0.9)$ & $5.7(0.8)$ \\
\hline \multicolumn{3}{|l|}{ Blood pressure $(\mathrm{mm} \mathrm{Hg})$ : } \\
\hline Systolic ${ }^{*}$ & $153.1(16.1)$ & $156.1(16.4)$ \\
\hline Diastolic* & $82.5(7.5)$ & $85.1(7.3)$ \\
\hline Variability in systolic $†$ & $14.8(5.0)$ & $13.9(4.6)$ \\
\hline Variability in diastolic $†$ & $7.1(2.9)$ & $7.4(2.3)$ \\
\hline \multicolumn{3}{|l|}{ Cognitive functionł: } \\
\hline Stroop test score (seconds) & $69.4(31.6)$ & $56.9(23.3)$ \\
\hline Letter-digit coding test score (digits coded) & $21.8(8.0)$ & $26.3(7.4)$ \\
\hline \multicolumn{3}{|l|}{ Picture-word learning test (pictures remembered): } \\
\hline Immediate recall score & $9.2(2.2)$ & $10.1(2.2)$ \\
\hline Delayed recall score & $9.8(3.1)$ & $11.1(3.0)$ \\
\hline \multicolumn{3}{|l|}{ MRI features: } \\
\hline Grey matter $(\mathrm{mL})$ & - & $590(44)$ \\
\hline White matter $(\mathrm{mL})$ & - & $768(38)$ \\
\hline Hippocampus (mL) & - & $7.5(1.1)$ \\
\hline No (\%) with microbleeds & - & $124(24.0)$ \\
\hline No (\%) with infarcts & - & $180(33.6)$ \\
\hline Cortical & - & $65(12.1)$ \\
\hline Lacunar & - & $112(21.0)$ \\
\hline White matter hyperintensity volume $(\mathrm{mL})$ & - & $7.2(1.1)$ \\
\hline
\end{tabular}

*Mean of all blood pressure measurements during follow-up.

†Standard deviation of all blood pressure measurements during follow-up.

$\ddagger$ Cognitive test score at end of follow-up. 
Table 2/ Cognitive function in thirds of visit-to-visit blood pressure variability. Values are means (standard errors) unless stated otherwise

\begin{tabular}{|c|c|c|c|c|}
\hline \multirow[t]{2}{*}{ Variables } & \multicolumn{3}{|c|}{ Third of visit-to-visit blood pressure variability } & \multirow[t]{2}{*}{$P$ value } \\
\hline & Low $(n=1820)$ & Middle $(n=1821)$ & High $(n=1820)$ & \\
\hline \multicolumn{5}{|l|}{ Systolic blood pressure } \\
\hline Range of SD (mm Hg) & $0.7-12.2$ & $12.3-16.2$ & $16.3-64.4$ & - \\
\hline Stroop test score (seconds) & $68.46(0.79)$ & $68.75(0.79)$ & $71.54(0.82)$ & $<0.001$ \\
\hline Letter-digit coding test score (digits coded) & $22.40(0.19)$ & $21.82(0.19)$ & $21.24(0.19)$ & $<0.001$ \\
\hline \multicolumn{5}{|l|}{$\begin{array}{l}\text { Picture-word learning test (pictures } \\
\text { remembered): }\end{array}$} \\
\hline Immediate recall score & $9.37(0.05)$ & $9.28(0.05)$ & $9.10(0.05)$ & $<0.001$ \\
\hline Delayed recall score & $10.00(0.07)$ & $9.89(0.07)$ & $9.70(0.08)$ & 0.001 \\
\hline \multicolumn{5}{|l|}{ Diastolic blood pressure } \\
\hline Range of SD $(\mathrm{mm} \mathrm{Hg})$ & $0-6.5$ & $6.6-8.5$ & $8.6-33.1$ & - \\
\hline Stroop test score (seconds) & $68.28(0.79)$ & $68.89(0.79)$ & $71.34(0.80)$ & $<0.001$ \\
\hline Letter-digit coding test score (digits coded) & $22.35(0.19)$ & $21.93(0.19)$ & $21.27(0.19)$ & $<0.001$ \\
\hline \multicolumn{5}{|l|}{$\begin{array}{l}\text { Picture-word learning test (pictures } \\
\text { remembered): }\end{array}$} \\
\hline Immediate recall score & $9.41(0.05)$ & $9.22(0.05)$ & $9.13(0.05)$ & $<0.001$ \\
\hline Delayed recall score & $10.01(0.07)$ & $9.88(0.07)$ & $9.74(0.07)$ & 0.001 \\
\hline
\end{tabular}

Analyses adjusted for age, sex, body mass index, statin treatment, apolipoprotein E genotype, country, education, test version where appropriate, smoking, cholesterol level, history of vascular diseases, history of hypertension, history of diabetes mellitus, and average blood pressure measures. 
Table 3/ Structural brain volumes in three groups of visit-to-visit blood pressure variability. Values are means (standard errors) (mL) unless stated otherwise

\begin{tabular}{|c|c|c|c|c|}
\hline \multirow[t]{2}{*}{ Variables } & \multicolumn{3}{|c|}{ Third of visit-to-visit blood pressure variability } & \multirow[t]{2}{*}{$P$ value } \\
\hline & Low & Middle & High & \\
\hline Systolic blood pressure & $(n=194)$ & $(n=210)$ & $(n=149)$ & \\
\hline Range of SD (mm Hg) & $0.7-12.2$ & $12.3-16.2$ & $16.3-64.4$ & - \\
\hline Grey matter & $593(3)$ & $590(3)$ & $589(3)$ & 0.21 \\
\hline White matter & $770(3)$ & $770(3)$ & $765(3)$ & 0.19 \\
\hline Hippocampus & $7.6(0.07)$ & $7.6(0.07)$ & $7.4(0.08)$ & 0.01 \\
\hline Diastolic blood pressure & $(n=178)$ & $(n=184)$ & $(n=191)$ & \\
\hline Range of SD (mm Hg) & $0-6.5$ & $6.6-8.5$ & $8.6-33.1$ & - \\
\hline Grey matter & $591(3)$ & $594(3)$ & $587(3)$ & 0.18 \\
\hline White matter & 768 (3) & $772(3)$ & $764(3)$ & 0.62 \\
\hline Hippocampus & $7.6(0.07)$ & $7.5(0.07)$ & $7.4(0.07)$ & 0.01 \\
\hline
\end{tabular}

Analyses adjusted for age, sex, body mass index, statin treatment, smoking, cholesterol level, history of vascular diseases, history of hypertension, history of diabetes mellitus, and average blood pressure measures. 
Table 4 | Microbleeds, infarcts, and white matter hyperintensities in three groups of visit-to-visit blood pressure variability. Values are odds ratios (95\% confidence intervals) unless stated otherwise

\begin{tabular}{|c|c|c|c|c|}
\hline \multirow[t]{2}{*}{ Variables } & \multicolumn{3}{|c|}{ Third of visit-to-visit blood pressure variability } & \multirow[t]{2}{*}{$P$ value } \\
\hline & Low & Middle & High & \\
\hline Systolic blood pressure & $(n=207)$ & $(\mathrm{n}=191)$ & $(n=137)$ & \\
\hline Range of SD (mm Hg) & $0.7-12.2$ & 12.3-16.2 & $16.3-64.4$ & - \\
\hline Microbleeds & 1 (ref) & $1.13(0.69$ to 1.85$)$ & $1.30(0.77$ to 2.21$)$ & 0.39 \\
\hline Infarcts & 1 (ref) & 0.95 (0.61 to 1.48$)$ & 1.26 (0.78 to 2.04$)$ & 0.40 \\
\hline Cortical & 1 (ref) & 1.34 (0.68 to 2.64$)$ & 2.22 (1.09 to 4.54$)$ & 0.02 \\
\hline Lacunar & 1 (ref) & $0.79(0.48$ to 1.31$)$ & $0.84(0.48$ to 1.46$)$ & 0.97 \\
\hline Mean (SE) WMH volume (mL) & $8.12(1.02)$ & $7.34(1.08)$ & $7.79(1.19)$ & 0.98 \\
\hline Diastolic blood pressure & $(\mathrm{n}=215)$ & $(\mathrm{n}=166)$ & $(n=154)$ & \\
\hline Range of SD (mm Hg) & $0-6.5$ & $6.6-8.5$ & $8.6-33.1$ & - \\
\hline Microbleeds & 1 (ref) & 1.75 (1.05 to 2.91$)$ & 1.77 (1.06 to 2.96$)$ & 0.01 \\
\hline Infarcts & 1 (ref) & 0.99 (0.63 to 1.56$)$ & 1.32 (0.84 to 2.06$)$ & 0.43 \\
\hline Cortical & 1 (ref) & 1.87 (0.93 to 3.76$)$ & 2.19 (1.10 to 4.37$)$ & 0.02 \\
\hline Lacunar & 1 (ref) & 0.95 (0.57 to 1.60$)$ & 1.17 (0.70 to 1.95$)$ & 0.75 \\
\hline Mean (SE) WMH volume (mL) & $7.65(1.05)$ & $8.27(1.11)$ & $7.93(1.10)$ & 0.55 \\
\hline
\end{tabular}

WMH=white matter hyperintensity.

Analyses were adjusted for age, sex, body mass index, statin treatment, smoking, cholesterol level, history of vascular diseases, history of hypertension, history of diabetes mellitus, and average blood pressure measures.

Data for microbleeds, infarcts, and white matter hyperintensities were available for 535 participants. 
Figure
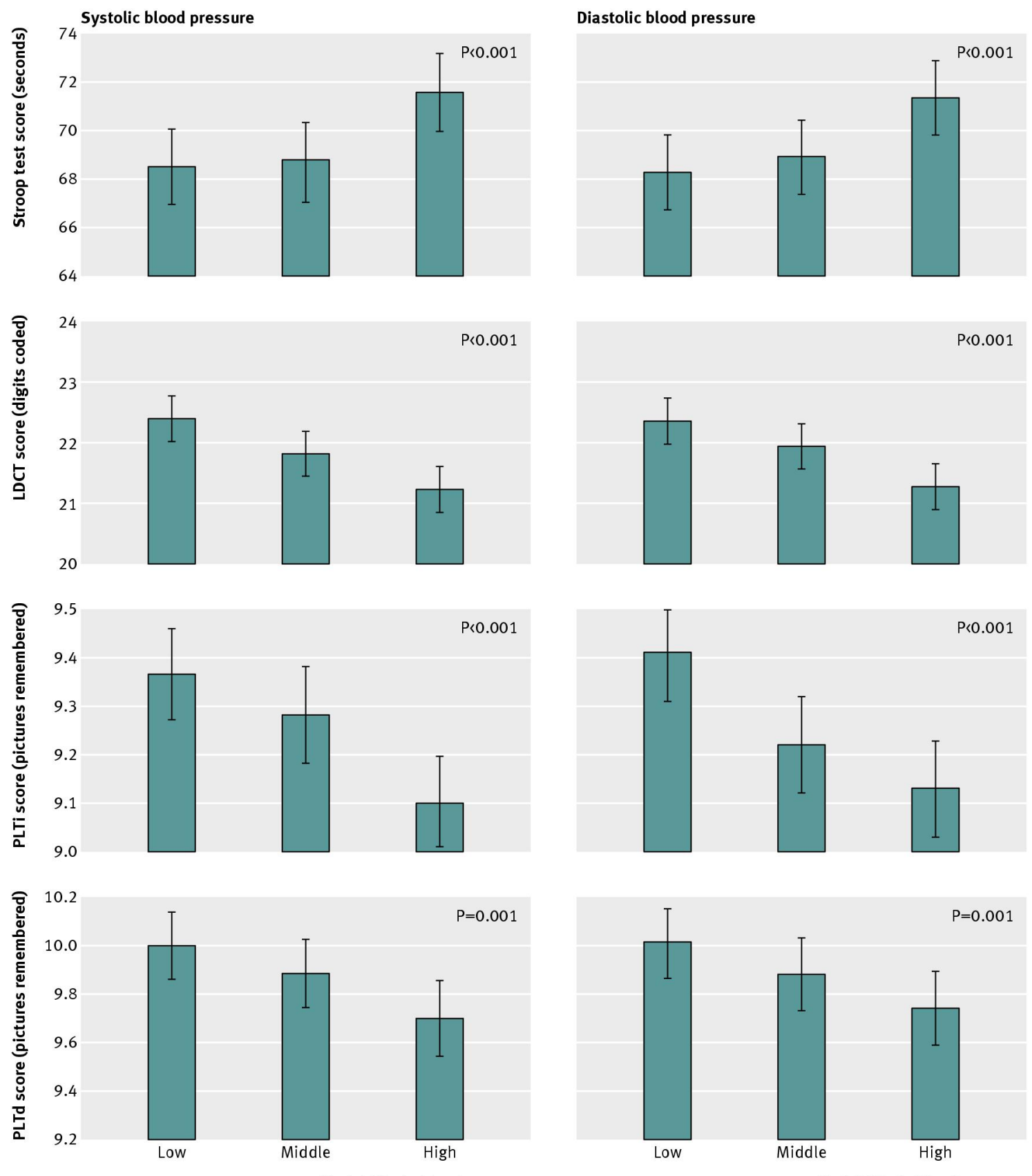

Variability in blood pressure

Variability in blood pressure

Scores for cognitive function in low, middle, and high thirds of variability in systolic and diastolic blood pressure between measurements. Bars represent means (95\% confidence intervals). Analyses were adjusted for age, sex, body mass index, statin treatment, apolipoprotein E genotype, country, education, test version where appropriate, smoking, cholesterol level, history of vascular diseases, history of hypertension, history of diabetes mellitus, and average blood pressure measures. LDCT=letter-digit coding test, PLTi=immediate picture-word learning test, PLTd=delayed picture-word learning test 